\title{
Las divergencias en torno a los humores en los compendios de medicina sánscrita
}

\author{
Juan Arnau Navarro (*) \\ $(*) \quad$ Instituto de Historia de la Medicina y de la Ciencia. CSIC-Universidad de Valencia \\ arnaujuan@gmail.com
}

Dynamis

[0211-9536] 2013; $33(2): 417-439$
Fecha de recepción: 5 de septiembre de 2012

Fecha de aceptación: 24 de febrero de 2013

SUMARIO: 1.-Introducción. 2.-Sustancias y sabores. 3.-Correspondencias humorales. 4.-Las divergencias en torno a los humores 5.-Asiento y funciones de los humores. 6.-Conclusiones.

RESUMEN: El artículo traza la genealogía de las divergencias sobre los humores en la literatura médica india del periodo clásico, utilizando como fuentes primarias las ediciones sánscritas de los compendios de Caraka, Suśruta y Vāgbhața. La tesis fundamental es sencilla, las diversas tradiciones médicas indias se desarrollaron en el marco de una teoría de los humores, pero las fuentes más antiguas de la tradición ayurvédica muestran que nunca hubo un acuerdo completo acerca de la naturaleza y funciones de los tres humores básicos (vata, pitta y kapha). En ocasiones los humores son considerados entidades físicas con una localización determinada en el organismo, en otras entidades hipotéticas para la clasificación de las enfermedades que deben ser equilibradas mediante el tratamiento médico. Esta ambigüedad en la definición, delimitación y aplicaciones de los humores dará lugar a diversos modos de entender el cuerpo humano y las diferentes terapias.

PALABRAS CLAVE: Ayurveda, medicina sánscrita, humores, Caraka, Suśruta, Vāgbhața.

KEY WORDS: Ayurveda, Sanskrit Medicine, Humours, Caraka, Suśruta, Vāgbhața.

\section{Introducción. Las fuentes: Caraka, Suśruta y Vāgbhața ${ }^{1}$}

El Compendio de Caraka (Caraka-saṃhitā) es la enciclopedia médica más antigua e influyente del Sur de Asia. Durante siglos ha servido de almacén

1. Sharma, Ram; Bhagwan, Dash, eds. Caraka Samhitā. Sanskrit text and critical exposition based on Cakrapāni Datta's Āyurveda Dīpikā. 7 vols. Varanasi: Chowkhamba Sanskrit Series Office; 1976-2001. Sharma, Priya, ed. Suśruta Saṃhitā, with Dalhana's commentary along with criti- 
de conocimientos, seguramente de diversa procedencia, de las diversas tradiciones médicas del subcontinente indio ${ }^{2}$. Encontramos referencias a esta obra tanto en las tradiciones filosóficas y literarias asociadas al brahmanismo, como en la literatura budista y en las crónicas de peregrinos chinos que recorrieron India en los primeros siglos de nuestra era. Tradicionalmente el Caraka ha sido el libro de texto de los estudiantes de medicina no sólo en India, sino también en Sri Lanka, Nepal o Birmania.

La obra contiene un total de 120 capítulos y está escrita en verso y en prosa. El verso utilizado (catorce diferentes tipos de métrica), es el clásico de la literatura sástrica, destinado a facilitar la síntesis y memorización de los contenidos. La enciclopedia se estructura en ocho secciones que cubren de manera desigual las diferentes especialidades médicas ${ }^{3}$. 1. Sütra (reglas generales). 2. Nidāna (etiología y diagnosis). 3. Vimāna (principios que gobiernan el cuerpo humano). 4. Śärîra (principios que gobiernan el nacimiento y la muerte). 5. Indriya (prognosis y síntomas). 6. Cikitsā (tratamientos y terapias). 7. Kalpa (farmacopea) y 8. Siddhi (conclusiones).

La autoría del Compendio de Caraka es incierta. La referencia externa más antigua al compilador de la obra se encuentra en el Samyukta-ratnapitakasūtra, texto budista de finales del siglo $\mathrm{V}$, donde se menciona al médico

cal notes. 3 vols. New Delhi: Chokhambha Orientalia; 1999-2001. Murthy, R. Srikantha, ed. Vāgbhațta's Aștaniga Hṛdayam. 3 vols. Varanasi: Chowkhamba Krishnadas Academy; 1991-1995. En adelante estas obras serán citadas con las abreviaturas CS: Caraka Samhitā, SS: Suśruta Sam hitā y AH: Astanga Hrdayam.

2. Desafortunadamente apenas se han investigado las diferentes capas históricas almacenadas en la obra, de modo que sigue siendo difícil determinar la fecha de cada pasaje en particular. El propio texto reconoce influencias de otras tradiciones médicas y menciona diferentes etnias y culturas del Sur de Asia, recomendando la prescripción de remedios en armonía con los hábitos y formas de vida de cada una de ellas. CS 6. 30, 315-20, p. 210-211. Se mencionan también pueblos distantes como persas, griegos, chinos y escitas, y es probable que algunos de los conocimientos de estas tradiciones fueran discutidos por los médicos indios, pero hay escasas evidencias en la propia obra que permitan su identificación. Sobre los paralelos con la medicina griega antigua: Filliozat, Jean. La doctrine classique de la médecine indienne. Ses origines et ses parallèles grecs. Paris: Imprimerie Nationale; 1949. Las investigaciones de Zysk han subrayado el papel de las tradiciones budistas en la preservación y transmisión del conocimiento médico: Zysk, Kenneth. Asceticism and healing in Ancient India: Medicine in the Buddhist monastery, Motilal Banarsidass: New Delhi; 1998. La propia obra confirma su carácter enciclopédico afirmando que «cualquier cosa que se menciona en este tratado se encuentra en el mundo, y lo que no se menciona no se encontrará en ninguna parte». CS 8. 12. 53-54, p. 447. Las traducciones son mías.

3. Sobre la taxonomía de las especialidades médicas: Majumdar, Ashok. Ayurveda: The Ancient Indian science of healing. New Delhi: Wheeler Publishing; 1998, p. 7. 
Caraka, consejero del rey Kanișka ${ }^{4}$. Otra de las referencias externas a la obra la encontramos en el Manuscrito Bower $(c a .600)^{5}$, donde se menciona el nombre de Caraka y donde se recogen varios fragmentos que tienen mucho en común con algunos pasajes de la obra, concretamente con el Navanitaka del Cikittā-sthāna. Respecto a las evidencias internas, el nombre de Caraka aparece al final de cada capítulo, donde se especifica el número y el título del mismo. La obra presenta sus contenidos como parte de la enseñanza del mítico sabio Ātreya a su discípulo Agniveśa (ambos legendarios y sin referencias históricas), presentando a Caraka como editor del compendio ${ }^{6}$.

Con todos estos datos y con las nuevas investigaciones sobre la preservación del conocimiento médico en ámbitos budistas, se considera que las primeras versiones de la obra pueden retrotraerse a los siglos III y II antes de nuestra era, aunque el texto no empieza a ser ampliamente citado en otros lugares de la literatura sánscrita hasta el periodo gupta (320-550). El consenso general es situar a Caraka en torno al siglo II, aunque algunos investigadores y pandits indios retrotraen su figura hasta el siglo VIII antes de nuestra era ${ }^{7}$. Un segundo editor posterior a Caraka, llamado Dṛdhabala (ca. 700), añadiría algunos pasajes y comentarios a la obra, que supuestamente le llegó incompleta. Dṛdhabala añadiría las porciones perdidas del texto y redactaría la sección séptima (Kalpasthānam) y la octava (Siddhisthānam) ${ }^{8}$, siendo posible que el texto completo fuera reescrito por él ${ }^{9}$. De entre los 43 comentarios sánscritos al Caraka mencionados en la literatura médica, sólo 8 han llegado hasta nosotros completa o parcialmente. De todos ellos destaca el comentario de Cakrapāni Datta (ca. 1000), Āyurvedadīpikā, que

4. Sobre la fecha de Kanișka: Basham, Arthur L., ed. Papers on the date of Kanișka. Leiden: Brill; 1968; y el trabajo del especialista en numismática del Museo Británico: Cribb, Joe. The early Kushan kings: new evidence for chronology. Evidence from Rabatak inscription of Kanishkal. In: Alram, Michael; Klimburg-Salte, Deborah, eds. Coins, art and chronology: Essays on the preIslamic history of the Indo-Iranian borderlands. Wien: Verlag der Österreichischen Akademie der Wissenschaften; 1999, p. 177-205.

5. Hoernle, Rudolf. The Bower manuscript. Facsimile leaves, Nagari transcript, Romanised transliteration and English translation with notes. New Delhi: Aditya Prakashan; 1987.

6. Sobre los orígenes míticos de la ciencia médica en India: Dash, Vaidya Bhagwan. Fundamentals of Ayurvedic medicine. Delhi: Sri Satguru Publications; 1999, p. 6-8.

7. Sharma; Dash, n. 1, p. xxxviii.

8. CS 6. 30. 289f, p. 200 y CS 8. 12. 36-40, p. 434-435.

9. Chattopadhyaya, Debiprasad. Science and society in Ancient India. Calcutta: Research India Publications; 1977, p. 31-33. 
la tradición considera el más autorizado ${ }^{10}$. Su autor parece plenamente consciente de los pasajes añadidos por Dṛdhabala y en numerosas ocasiones justifica la organización del texto y ofrece lecturas alternativas a erratas y lagunas. En el siglo XI la obra ya es bien conocida en el mundo islámico. El nombre de Caraka aparece citado en las traducciones latinas de la obra de Abū ibn Sīnā (Avicena) y también en las obra de Abū Rayhān al-Bīrūnī (Alberuni) e Ibn Sarabi.

La segunda de las fuentes primarias que utilizaremos en este artículo es La esencia [de la ciencia] óctuple (Așțàngahṛdaya) de Vāgbhața (ca. 600), traducido con frecuencia como La esencia de la medicina, por estar ésta constituida de ocho especialidades, es probablemente la obra médica más leída en el subcontinente indio. Su gran capacidad de síntesis, su claridad expositiva, su enorme difusión y popularidad en las diversas tradiciones del ayurveda, su utilización como libro de texto para la formación de médicos y sus numerosos comentarios, referencias y alusiones, la convierten en una de las tres obras fundamentales de la literatura ayurvédica, junto a los compendios, más antiguos, de Caraka y Suśruta ${ }^{11}$. Incluso hoy se pueden encontrar, en algunas familias de Kerala, médicos que han memorizado extensos fragmentos de la obra.

La obra se reconoce a sí misma como síntesis, «ni muy sucinta ni muy elaborada» de los principales tratados médicos de la antigüedad ${ }^{12}$. El contexto histórico de las obras de Vāgbhața pertenece a las postrimerías del periodo Gupta, probablemente La esencia fue compuesta en torno a los inicios del siglo VII ${ }^{13}$. Sobre la identidad de su autor apenas sabemos nada, únicamente lo que él mismo menciona en el colofón de otra de sus obras, el Compendio de la medicina (Aștāngasangraha): «Hubo una gran médico llamado Vāgbhața que fue mi abuelo y del que llevo el nombre. De él nació Simhagupta, mi padre. Nací en el país del Sind y aprendí la ciencia

10. Cakrapāni además suministra nombres coloquiales o vernáculos para la farmacopea sánscrita. El Caraka fue traducido al árabe en el siglo VIII y difundido entre los médicos persas.

11. Wujastyk, Dominik. The roots of Ayurveda. Selections from Sanskrit medical writings. London: Penguin; 2003, p. 193. Wujastyk compara su importancia en el Sur de Asia con la que tuvo el Canon de medicina de Avicena en Europa y Medio Oriente. Del mismo modo que Avicena ordenó y dio unidad al enorme corpus de textos dejados por Galeno, Vāgbhața sistematizó y dio coherencia y claridad a los extensos compendios de Caraka y Suśruta.

12. $A H$ 1. 1. 4, p. 8 .

13. Meulenbeld, Gerrit Jan. A history of Indian medical literature. 5 vols. Groningen: E. Forsten; 1999-2002, vol. 1, p. 597-656. 
del ayurveda de Avalokita, mi preceptor, y sobre todo de mi padre. Tras estudiar un gran número de textos de dicha ciencia, redacté el presente tratado» ${ }^{14}$. A pesar de la popularidad de La esencia en el Sur de India, Meulenbeld apunta que lo más probable es que su autor fuera originario del Sind (al norte de la actual Karachi).

Es posible que el propio Vāgbhața fuera educado en el brahmanismo y con el paso del tiempo adoptara la fe budista. Los que sostienen esta opinión aluden a que el nombre de su preceptor, Avalokita, es un nombre budista y que otros nombres que se mencionan en sus obras tienen también este origen. Otra de las pruebas que justificarían esta opinión es el hecho de que el Buda es mencionado explícitamente en el epígrafe de invocación del Aștāñgasañgraha y, de manera indirecta, en el de La esencia ${ }^{15}$. Para algunos investigadores estas pruebas no son concluyentes y la cuestión sigue abierta.

De los contenidos de su obra podemos deducir que Vāgbhața dominaba los compendios de Caraka y Suśruta y que su propósito fue unificarlos y ofrecer una síntesis comprensible y de fácil lectura de estas dos obras monumentales. El trabajo es una muestra acabada de la rica tradición médica india, estructurada de forma que se hacen fácilmente accesibles sus contenidos. Otra obra atribuida a Vāgbhața es el Aștāñgasañgraha (Compilación [de la ciencia] óctuple), aunque durante un tiempo se consideraron obras de diferentes autores con un mismo nombre, hoy día se considera que se trata de una obra posterior del propio Vāgbhața. Ambas tienen mucho en común, y lo que en La esencia se dice en verso, en La compilación aparece en prosa, lo que facilita en muchos casos su comprensión.

Las fuentes primarias del presente artículo se limitan a las enciclopedias médicas sánscritas del periodo clásico de Caraka $(C S)$ y Vāgbha.ta $(A H)$, con alguna referencia ocasional a Suśruta $(S S)$, así como al comentario medieval de Cakrapānịi. Aunque estas obras difieren en el análisis de la constitución y en la determinación de las funciones de los humores, todas ellas coinciden en listar tres humores básicos: aire (vāta), bilis (pitta) y flema (kapha). En general se considera que los humores fluyen a través del cuerpo impregnando completamente el organismo y que su naturaleza ambivalente los capacita tanto para el mantenimiento de la vida como pa-

\footnotetext{
14. AS 6. 50, p. 203-204.

15. Sobre las prácticas médicas y la educación médica en los monasterios budistas: Zysk, n. 2, p. 38-49.
} 
ra su destrucción ${ }^{16}$. La mezcla regular de los humores garantiza la salud, mientras que su corrupción origina las llamadas enfermedades internas ${ }^{17}$. La enfermedad se entiende como un desequilibrio de los humores, sin que ello impida que pueda haber otras enfermedades que no dependan de ellos. ${ }^{18}$ Esta taxonomía determina tanto la farmacología como los tratamientos, clasificando medicamentos y terapias en dos tipos: paliativos (śamana) y purificativos (śodhana). Los primeros se destinan a mitigar la agravación de los humores, mientras que los segundos eliminan humores exasperados ${ }^{19}$.

La consideración de los humores como el factor determinante tanto de la salud como de la enfermedad y su relación con la vida y experiencias mentales del paciente tiene como consecuencia que, de manera general, los tratamientos físicos se complementen con ciertas terapias mentales como la discriminación $(d h \bar{\imath})$, la determinación (dhairya) y el autoconocimiento (ätmädivijñāna). La primera permite discriminar lo bueno de lo perjudicial, la segunda es la disposición a adherirse a lo beneficioso, la tercera permite determinar los objetivos tanto de la vida presente como de la futura. En este sentido la antropología filosófica jugará un papel fundamental en la ciencia médica ${ }^{20}$. Cuatro son los factores que determinarán el éxito o el

16. AH 1. 11, 34-45, p. 163-165.

17. «Del mismo modo que el pájaro que vuela a cielo abierto no puede evitar su sombra, así, los diferentes tipos de enfermedades no pueden existir sin los humores (doșa), incluso la corrupción de los tejidos (dhātu) no puede entenderse sin la implicación de éstos». AH 1. 12. 32-34, p. 173.

18. A las enfermedades humorales se las denomina nija, porque surgen del propio organismo, mientras que las debidas a causas externas, fundamentalmente accidentes, se las llama ägāntu. AH 1. 20. Además, las enciclopedias distinguen entre los humores del cuerpo (śärïrakadoșa) y los humores de la mente (mänasadoșa).

19. La administración de diferentes tipos de enemas suele ser el método recomendado para mitigar vāta, las purgas para pitta y los vómitos para kapha. El uso de aceites medicinales, tanto interna como externamente, se recomienda para atenuar vāta, mientras que el ghee (ghrta) se utiliza para pitta y la miel para kapha.

20. Nos referimos a los sistemas filosóficos sāmkhya y vaiśenika, dos de los seis sistemas ortodoxos del brahmanismo, que muy probablemente ejercieron su influencia en las disciplinas médicas de la época clásica. Son numerosos los ejemplos en los cuales «los conceptos médicos se ajustan a las ideas filosóficas». Meulenbeld, Jan. Reflections on the basic concepts of Indian pharmacology. In: Meulenbeld, Jan; Wujastyk, Dominik, eds. Studies on Indian medical history. Groningen: Forsten; 1999, p. 1-17 (4). Un buen estudio que analiza dicha injerencia, con relación al sistema vaiśeniika es el de Comba, Antonella. Carakasaṃhitā, Śārīrasthāna I and Vaiśenika philosophy. In: Meulenbeld, Jan; Wujastyk, Dominik, eds. Studies on Indian medical history. Groningen: Forsten; 1999, p. 39-52. Ciertos tratamientos agresivos (como los destinados a la quema de tejidos) que acarrean un deterioro de la experiencia mental, no serán aceptados por el ayurveda, que se inscribe en un marco de creencias donde la 
fracaso de un tratamiento: el paciente, el médico, el enfermero y la medicación. Para cada uno de ellos se listan toda una serie de cualidades. Del médico se exige experiencia y erudición, de la droga potencia, del enfermero paciencia y afecto, y del paciente obediencia, voluntad y memoria. Otros factores condicionarán la estrategia en cualquier tipo de curación: la dieta, el hábitat (deśa), la estación del año $(r+u)$, la constitución del cuerpo (prakrti) y la configuración de los planetas.

Sin embargo, los humores no son los únicos factores internos que determinan el estado general del organismo, hay otros elementos a tener en cuenta, fundamentalmente los tejidos (dhätu) y los desechos (mala). Los tejidos, que también se denominan $d \bar{u} s y \bar{a}$ («aquellos corrompidos por las doṣa»), se listan siete básicos: plasma (rasa), sangre (aşrk), músculos (māmsa), grasa (medas), huesos (asthi), médula (majja) y semen (śukra). Los desechos (mala) fundamentales son la orina (mūtra), las heces (śakrt) y el sudor $(\text { sveda })^{21}$.

El delicado equilibrio de estos tres factores: humores, tejidos y desechos (doșa, dhätu y mala), constituye la base de la salud. El incremento o disminución de la actividad humoral se regula mediante el uso de sustancias similares (samāna) o contrarias (viparìta) a la esencia de los diferentes doṣa. Cada uno de ellos tiene su justa medida (cantidad, cualidad y actividad) y cuando este equilibrio se rompe se inician los procesos mórbidos. Según La esencia, la ciencia médica se ocupará de identificar y prescribir la utilización de sustancias que puedan potenciar o contrarrestar los incrementos o disminuciones de estos tres factores básicos del organismo. Esto nos lleva a la noción de sustancia (dravya).

\section{Sustancias y sabores}

Una de las taxonomías de las sustancias en la literatura médica establece tres tipos generales: aquellas que alivian los humores (śamana), aquellas que los agravan (kopanā) y aquellas que mantienen su equilibrio (svasthahita) ${ }^{22}$. Ya se trate de alimentos o de medicinas, las sustancias tienen diferentes

reencarnación es una posibilidad real y el estado de la mente en el tránsito de una vida a otra resulta decisivo.

21. $A H$ 1. 13. 1-41, p. 182-190.

22. $A H$ 1. 16. 1-46, p. 208-218. 
sabores (rasa), que se listan en seis: dulce ( $s v \bar{a} d u)$, ácido (amla), salado (lavana), amargo (tikta), acre o picante (üsana) y astringente (kasāya). Estos sabores se encuentran presentes en mayor o menor medida en todas las sustancias, de ahí que se les considere una cualidad ( guna) de las mismas y al mismo tiempo un principio activo (karman). Además, dichos «sabores» pueden analizarse en función de los elementos (mahäbhüta) que las componen. Estos elementos son cinco: éter (ākáśa), aire (vāyu), fuego (agni), agua $(\bar{a} p a h)$ y tierra $(p r t h i v \bar{a})$. Las desavenencias entre los compendios se hacen ya manifiestas en este caso. Caraka considera las sustancias dulces compuestas predominantemente por agua, mientras que para Suśruta y Vāgbhața por agua y tierra ${ }^{23}$.

Además, cada sustancia puede tener uno o más sabores, que se ponen de manifiesto al entrar en contacto con la lengua. El sabor primero y claramente reconocible de una sustancia es el pradhänarasa, mientras que los sabores secundarios se denominan anurasa. La importancia de los rasa en el tratamiento médico radica en que éstos guardan una estrecha relación con los humores. Lo dulce, ácido y salado alivian o disminuyen vāta. Lo amargo, picante y astringente disminuyen kapha, mientras que lo dulce, lo astringente y amargo reducen pitta. La ingestión de sustancias con sabores diferentes a los prescritos en las correlaciones anteriores producirá el incremento ( $v r d d h i)$ de la actividad de los diferentes humores. Es decir, lo amargo, lo picante y astringente incrementa vāta, lo ácido, salado y picante incrementa pitta, mientras que lo dulce, lo amargo y salado incrementará la actividad de kapha. Vāgbhața dedica el capítulo décimo de La esencia a detallar los beneficios y perjuicios de los diferentes sabores, que merecen por sí mismos un artículo completo ${ }^{24}$. Otro de los factores determinantes de las sustancias es su potencia (virya), responsable de las transformaciones que puedan producir en el organismo. La potencia puede ser de dos clases: caliente (usna) y fría (śita ${ }^{25}$. Pese a todo, la historiografía reciente ha mos-

23. Meulenbeld, n. 20, p. 5. Para Meulenbeld la doctrina de los sabores forma parte de un antiguo corpus de textos de las tradiciones médicas mientras que los intentos de interpretarlos en función de los elementos pertenecen a un estrato posterior.

24. El Caraka es claro al respecto: "La alimentación sostiene la vida de los seres. La complexión, la claridad, la buena voz, la longevidad, el genio, la felicidad, la satisfacción, la nutrición, la fuerza y el intelecto están condicionados por el alimento». CS 1. 27. 349, p. 564.

25. Hay además otro factor, vipäka, el producto final de la digestión, que puede ser dulce, amargo o acre. AH 1. 17. 1-30, p. 219-224. 
trado que, a pesar de los esfuerzos de los comentaristas medievales, la teoría de los sabores se encuentra lejos de estar unificada en el periodo clásico ${ }^{26}$.

\section{Correspondencias humorales}

Lo primero que se percibe en un análisis cuidadoso del concepto de «humor» en los tres compendios clásicos de medicina sánscrita es que se trata de una entidad ambigua y que en muchos casos los compiladores evitan definir con precisión. Para una primera aproximación quizá sea conveniente examinar sus relaciones con otros elementos del mundo natural. Dentro del marco de correspondencias entre lo macrocósmico y lo microcósmico, los humores (vāyu, pitta y kapha), cuya función es mantener la vida del organismo, se entienden también como «condensaciones activas» y relativamente inestables de los cinco elementos físicos: espacio, aire, fuego, agua y tierra ${ }^{27}$. «En la visión del mundo india la persona es vista como un microcosmos; todo lo que es parte del cosmos tiene su homólogo dentro de la persona ${ }^{28}$. El primero de los humores, $v \bar{a} y u$, se compone principalmente de espacio y viento, el segundo, pitta, de fuego y agua, y el tercero, kapha, de agua y tierra. Todas las sustancias del mundo natural están hechas de estos cinco elementos, pero desde la perspectiva e intereses de la ciencia médica, resulta más útil hablar de humores que de elementos.

El primero de los humores, vāyu o vāta (aire) es responsable de todo el movimiento que se produce en el cuerpo y en la mente. Ya se trate de un órgano, un alimento o un recuerdo, todo lo que se mueve en el organismo se mueve gracias a vāyu. El segundo de ellos, pitta (bilis), se ocupa de todas las transformaciones que se producen en el cuerpo, digiere la comida, pero también digiere la experiencia sensible, afectiva e intelectual, lo que vemos, lo que amamos y lo que aprendemos. El tercero de los humores, kapha o śleșman (flema o mucosidad), representa la estabilidad, lubrica-

26. Meulenbeld, n. 20, p. 16.

27. Svoboda, Robert. Theory and practice of Ayurvedic medicine. In: Van Alpen, Jan; Aris, Anthony, eds. Oriental Medicine. London: Serindia Publications; 1995, p. 67-97 (74).

28. Kakar, Sudhir. Shamans, mystics and doctors: A psichological inquiry into India and its healing traditions. London: Unwin; 1984, p. 362. La equivalencia entre el hombre y la naturaleza, entre los componentes físicos de la persona y los del cosmos se encuentra en las cinco formas radicales de la materia (pañcabhüta) y constituye una piedra angular de conocimiento ayurvédico. CS 4. 5. 2-5, p. 414. 
ción y equilibrio del organismo, frenando los excesos de los anteriores. Los tres humores son obviamente complementarios y la vida orgánica no podría entenderse sin su actividad dinámica, metabólica y estabilizadora. Mientras las energías internas fluyan adecuadamente la enfermedad se mantendrá alejada.

Los humores son esencialmente ambiguos. Algunos pasajes subrayan la importancia del equilibrio humoral, mientras que otros ponen el énfasis en su correcta localización. Ya hemos mencionado que a veces se conciben como entidades fluidas que recorren el organismo, manteniendo su vigor, y otras como responsables de los procesos morbosos que afectan al organismo, produciendo la enfermedad cuando se acumulan o ausentan de ciertos lugares. Es por ello que son denominados doșa, palabra que en su sentido habitual (no técnico) significa defecto, imperfección, tara, y cuyo significado, en un sentido moral, se extiende a todo aquello perjudicial para el espíritu y la moral. De hecho, el término sánscrito procede de la raíz duș, equivalente al prefijo español dis (disfunción, discapacidad). En su justa medida o estado normal (svamāna), los humores mantienen el cuerpo, y lo recomendable es conservarlos en dicho estado ${ }^{29}$ pero cuando se vician producen los síntomas de la enfermedad, y pueden llegar a colapsar el organismo.

El juego de las correspondencias, tan grato a la literatura médica y filosófica de la antigüedad india, no se detiene aquí. Los humores se relacionan además con las etapas de la vida, los momentos del día y las fases de la digestión ${ }^{30}$. Respecto a la actividad humoral, el día es una vida en miniatura. Por otro lado, la digestión es un proceso metabólico que corre en paralelo al recorrido del Sol. Caraka y Vāgbhața difieren en sus asignaciones. Para La esencia, vāta predomina al atardecer (entre las tres y las siete de la tarde) y entrada la noche (desde las dos a las seis de la madrugada), así como en la fase final de la digestión. Vāta es también el humor dominante en la vejez (a partir de los sesenta años). Pitta, por otro lado, es especialmente influyente en la madurez (desde los veinte a los sesenta años), al mediodía (entre las once y las cuatro), y en el periodo medio de la digestión. Kapha domina en la infancia, el atardecer (desde las seis hasta las diez), y en las primeras 
fases de la digestión ${ }^{31}$. El Caraka invierte la relación entre los humores y las etapas de la vida, vāta predomina en la infancia y kapha en la vejez ${ }^{32}$.

Finalmente, los humores son también responsables del logro de los objetivos fundamentales de la vida, los llamados puruṣārtha. No sólo contribuyen a hacer posible la actividad sensorial, el vigor físico, la dicha y la longevidad, sino que, bien orientados, hacen posible el placer, la virtud, la riqueza y la autorrealización. La felicidad de aquí y la del más allá ${ }^{33}$.

\section{Las divergencias sobre los humores}

La ambigüedad esencial de los humores respecto a su presencia y funciones dentro del organismo se extiende también a sus posibles interpretaciones. Cuando se analizan los marcos teóricos del ayurveda, con frecuencia se habla de la doctrina de los humores como una teoría aceptada y consolidada desde la antigüedad. Pero lo cierto es que, si examinamos lo que dicen los textos, en seguida advertimos que, lejos de constituir un marco teórico definitivo para el ejercicio de la práctica médica, la teoría humoral ha sido objeto de diversas interpretaciones y, a pesar de los esfuerzos de Vāgbhața por establecer una «teoría unificada» (fundamentalmente mediante la conciliación de las visiones de Caraka y Suśruta), las divergencias permanecieron. Como apunta Meulenbeld, Caraka y Suśruta difieren en un número considerable de cuestiones, razón por la cual el sistema ayurvédico en la época clásica no puede considerarse un sistema cerrado y los comentaristas posteriores de los compendios se esforzarán en limar las diferencias ${ }^{34}$. En ocasiones no es claro si los humores se consideran como entidades físicas o simplemente como manifestaciones orgánicas que se hacen visibles por sus efectos, pero que carecen de un sustrato físico identificable, siendo entonces lícito considerarlos como un «estado de ánimo» de los tejidos.

El propio Caraka no elude la cuestión y se pregunta si se trata de entidades hipotéticas que sirven para agrupar los diferentes tipos de enfermedades y dolencias o si deben considerarse como entidades físicas, semisustancias fluidas que se distribuyen por todo el organismo y tienen

31. AH 1.7. 7, p. 110.

32. CS 4. 6. 20, p. 441.

33. CS 1. 12. 13, p. 242.

34. Meulenbeld, n. 20, p. 3. 
sus lugares propios de concentración. Cuando se encuentra con un conjunto particular de síntomas, el médico identifica la perturbación de uno de los humores. Pero, ¿son éstos entidades materiales segregadas por el organismo? En algunos pasajes Caraka considera los humores como constituyentes del cuerpo (dhātu) y residuos (mala), y en otros lugares como factores que afectan a éstos, que en proporciones adecuadas sostienen el edificio del cuerpo y que, desequilibradas, lo arruinan. «Los tres doṣa están presentes en el cuerpo de todas las criaturas. El médico tendrá que distinguir si se encuentran en un estado normal o mórbido» ${ }^{35}$.

Este hecho queda confirmado al analizar los procesos de asimilación y evacuación corporal. La esencia de lo que se asimila mediante la digestión del alimento y la bebida se denomina rasa (jugo), mientras que lo que se deshecha se denomina kitța o mala, término que hace referencia a los diversos tipos de excreción corporal. Los residuos fundamentales son el sudor, la orina y el excremento, pero hay otros como la cera, las legañas o la mucosidad, y los humores (vãyu, pitta y kapha) que también se eliminan $^{36}$. De modo que los humores se consideran también como secreciones del cuerpo, y éstas, en su justa medida, contribuirán como los humores a su mantenimiento. De todas las secreciones estas tres, vāyu, pitta y kapha, son para Caraka las más importantes, pues constituyen el fundamento del crecimiento y decadencia del cuerpo.

Las discordancias respecto a la funcionalidad de los humores se hacen evidentes en las diferentes taxonomías que ofrecen las enciclopedias. En general cada uno de los humores se subdivide en cinco tipos, de acuerdo con las diferentes funciones que realizan, sin embargo, esta subdivisión no aparece en el Caraka respecto a pitta y kapha. Suśruta describe los cinco tipos de vāyu y de pitta, pero al explicar las clases de kapha no utiliza la misma terminología que encontramos en Vāgbhața y en los compendios posteriores $^{37}$.

Zysk ha mostrado la continuidad entre la medicina budista, practicada en los monasterios y registrada en los códigos de conducta (vinaya), y la medicina ayurvédica consignada en el compendio de Caraka ${ }^{38}$. Según el

\footnotetext{
35. CS 1. 18. 48, p. 346.

36. CS 1. 28. 3, p. 566.

37. Una tabla sintética de estas funciones se encuentra en Majumdar, n. 3, p. 58.

38. Zysk, Kenneth. Asceticism and healing in Ancient India: Medicine in the buddhist monastery. New Delhi: Motilal Banarsidas; 1998.
} 
vinaya, tanto vāyu, pitta o kapha, como una combinación de estos, pueden ser causa de enfermedades, aunque existen casos en que las dolencias no tienen que ver con éstos, como por ejemplo en las enfermedades estacionales. En estos casos, los doṣa parecen más bien referirse a algo que no debiera estar normalmente en el cuerpo, un «defecto», consecuencia de las condiciones anormales de vāyu, pitta y kapha. Y, como apunta Scharfe, esto se encuentra lejos todavía de lo que usualmente se entiende por «etiología humoral» ${ }^{39}$. En la colección budista del Dighanikāya, por ejemplo, encontramos una lista en la que pitta y kapha son considerados parte del cuerpo humano (junto con la orina o las heces no evacuadas), mientras que vāyu claramente no forma parte del mismo. En textos budistas como el Buddhacarita o el Manuscrito Bower encontramos referencias a un «trío de defectos» (doṣa-traya), un trío de elementos (dhätu-tritaya) y la buena salud se define como un equilibrio de estos últimos. Los dhātu están serenos cuando los doșa son eliminados o apartados, y esos doṣa aparecen como estados, supuestamente alterados, de vāyu, pitta y kapha. Aunque según la doctrina clásica del ayurveda la salud de la persona depende del equilibrio de los tres doṣa, un análisis genealógico del término señala a la influencia de los sistemas filosóficos en las tradiciones médicas. Scharfe ha mostrado de manera convincente que, en sus primeras manifestaciones, los doṣa se refieren a ciertas irregularidades que producen la enfermedad y más tarde, bajo la influencia del sāmkhya, pasan a convertirse en una característica necesaria de la buena salud, en paralelo con las tres «virtudes» $(\text { gun } a)^{40}$.

Los diversos enfoques se sucederán a lo largo de la literatura de comentarios hasta el periodo colonial y la llegada de la medicina europea. En general sólo en textos posteriores a la invasión musulmana (siglo XI), que introdujo en el subcontinente la medicina unani (versión persa del galenismo), se habla de un cuarto humor, la sangre, pero a lo largo del periodo clásico, salvo en el caso de Suśruta, los humores en la literatura ayurvédica serían estos tres. Suśruta es una notable excepción y el papel ambiguo de la sangre, a veces citada entre los doṣa y otras entre los dhätu, ha sido objeto de numerosos comentarios por parte de compiladores y editores de textos

39. Scharfe, Hartmut. The doctrine of the three humors in traditional Indian medicine and the alleged antiquity of Tamil Siddha medicine. Journal of the American Oriental Society 119 (4); 1999: 609-629 (614).

40. Scharfe, n. 39, p. 629. 
ayurvédicos ${ }^{41}$. Como señala Meulenbeld, la incorporación de la sangre como un cuarto humor constituye un tema decisivo que apunta a un periodo de la historia de la medicina india en el que el rígido dogmatismo de los tres humores no se había producido ${ }^{42}$.

Por ejemplo, mientras Vāgbhața diferencia claramente entre constituyentes corporales $(d h \bar{a} t u)$, humores $(d o s ̦ a)$ y residuos $(m a l a)^{43}$, en Caraka encontramos que, en función de su comportamiento, los humores pueden manifestarse o bien como constituyentes corporales o bien como residuos. En La esencia, Vāgbhața niega que los tejidos o los desechos puedan ser, en sí mismos, causa de enfermedades, calificando otras interpretaciones de «figuradas». Para Vāgbhața, el cuerpo humano es la unión de estos tres elementos: dhätu, doșa y mala, y en modo alguno pueden resultar intercambiables a consecuencia de las funciones que realizan en el cuerpo ${ }^{44}$.

Algunos comentaristas posteriores a Vāgbhața considerarán los humores como sustancias que dinamizan y mueven los tejidos (dhätu), y que la circulación de sustancias semifluidas a través del organismo se debe a ellos. La distinción entre lo que corrompe y lo corrompido parece mantenerse. A la primera categoría pertenecen los doṣa, a la segunda los dhätu. La enfermedad no se considera la desproporción o irregularidad (vaișamya) de los dhātu sino de los doșa, considerando a ambas entidades independientes, de modo que una perturbación en los doṣa no implica necesariamente una perturbación de los dhātu ${ }^{45}$.

41. Meulenbeld, Gerrit Jan. The constraints of theory in the evolution of nosological clasifications: A study on the position of blood in Indian medicine (Ayurveda). In: Meulenbeld, Gerrit Jan, ed. Medical literature from India, Sri Lanka and Tibet. Leiden: Brill; 1991, p. 91-106 (91).

42. Meulenbeld, n. 20, p. 3.

43. Vāyu sostiene la energía del cuerpo, la respiración y el movimiento mental y corporal; pitta se ocupa de las funciones digestivas, la vista, el coraje, la imaginación y la capacidad de comprensión y śleniman de la firmeza y suavidad de las articulaciones.

44. AH 1. 11. 1-36, p. 154-163.

45. Las distintas filosofías y distintas concepciones de la causalidad han servido de trasfondo al pensamiento médico, que influirán en la concepción de la enfermedad y su relación con los humores. Comba, n. 20, p. 39-44. Para el sāmnkhya, que sostiene que el efecto es inherente a la causa, el desequilibrio de los doșa y la enfermedad constituirán un mismo fenómeno. Sin embargo, desde la perspectiva nyāya, que sostiene que el efecto es diferente de la causa, la enfermedad no es en sí misma «humoral», aunque sea producida por los humores, diferenciando así la enfermedad de sus síntomas. Vāgbhața parece inclinarse por la primera opción, y en su Compendio médico (Aștangasangraha 1.22.) recurre a una metáfora acuática. Al igual que las olas, con sus diferentes tamaños, crestas y espumas, pertenecen todas al 
En esta línea de pensamiento hay una excepción en el periodo clásico. Suśruta parece otorgar a la sangre (śonita) un mismo estatus que a vāta, pitta y kapha. Ello se debe a que El compendio de Suśruta es fundamentalmente un tratado de cirugía, donde la sangre y sus infecciones juegan un papel fundamental en el mismo. Suśruta asume la localización tradicional de los humores ofrecida por Caraka, pero añade que la sangre realiza funciones similares a éstos en el mantenimiento o decaimiento del organismo ${ }^{46}$. El tratado también ahonda en el juego de las correspondencias entre los humores y los elementos cósmicos como el aire, el fuego y el agua ${ }^{47}$. Todas estas consideraciones reafirman la idea de que los doṣa son considerados agentes materiales, por ser de alguna manera derivaciones de los dhātu y, originalmente, de los mahäbhūta. Esta idea será desmentida posteriormente por Vāgbhața, que se inclina a considerarlos como entidades abstractas (o al menos no específicamente materiales) que permean todas las partes del cuerpo con diversa intensidad y que sirven de etiquetas para la clasificación de toda una serie de síntomas y enfermedades ${ }^{48}$.

Caraka admite que algunos médicos consideran inviable el completo equilibrio de vāyu, pitta y kapha en los seres vivos. Semejante equilibrio debe considerarse más un estado ideal que una realidad empírica. Al margen de la constitución individual de cada uno, que ya supone cierto desequilibrio humoral interno, por mucho que se cuide la alimentación y la experiencia mental y sensible (que también se digiere), ésta adolecerá siempre de cierto grado de descompensación. Ello conducirá lógicamente a la posibilidad de establecer arquetipos humanos de origen humoral ${ }^{49}$.

Se trata de una cuestión de actitud y disposición, que orienta la personalidad hacia determinados ámbitos de la experiencia. Este proceso se inicia ya en el desarrollo del embrión, dando lugar, de manera general, a tres clases de individuos. Las personas dominadas por kapha (flemáticas) son generalmente saludables, mientras que en las dominadas por los otros dos

océano; así las enfermedades, con sus diferentes síntomas y naturalezas, pertenecen todas a los humores (doșa).

46. SS 1. 5. 12, p. 186.

47. SS 3. 4. 80, p. 205.

48. Dash (n. 6, p. 21-25) ofrece una lista muy completa de terminología sánscrita referida a síntomas y enfermedades agrupados por el agravamiento de cada uno de los humores en particular.

49. Caraka pasa a describir los individuos cuyo humor preponderante es vāta, llamados vātala, los pittala y los ślenmala, junto con los tratamientos que compensen su inclinación natural en CS 3. 6. 15-19, p. 190. Para los aspectos psicológicos de los humores: Majumdar, n. 3, p. 55. 
humores la salud dependerá de otros factores. Para Caraka, sin embargo, esto contradice un hecho fundamental: el estado natural del cuerpo es el estado saludable. Efectivamente, los individuos que se encuentran dominados por alguno de los humores no pueden considerarse en su «estado normal o natural». De hecho ese equilibrio, aunque nunca sea perfecto, debe considerarse el punto de partida de los que llamamos salud ${ }^{50}$. La definición de lo natural experimenta aquí ciertas tensiones. La cuestión es si concebimos lo natural como una condición más o menos mórbida o como un estado saludable.

La idea de los humores de Vāgbhața, la más difundida y universalmente reconocida en la tradición ayurvédica, supone un esfuerzo de síntesis y conciliación de las concepciones de Caraka y Suśruta. La esencia dedica el apartado once del primer capítulo a la «ciencia de los humores». Según Vāgbhața, los componentes fundamentales del cuerpo son de tres tipos: humores $(d o s a)$, tejidos corporales (dhätu) y deshechos (mala). El primero de los humores, $v \bar{a} y u$ o vāta (viento, aire), en su estado natural, ayuda a mantener la energía física, la respiración y las necesidades naturales del cuerpo. Contribuye también al movimiento de los tejidos corporales y la agudeza de la percepción. El segundo, pitta (bilis o cólera), ayuda con la digestión, el calor corporal y la visión. También participa del hambre y la sed, del apetito, el buen aspecto, la memoria, la inteligencia, el coraje y la flexibilidad. El tercero, kapha (flema o mucosidad), contribuye a la firmeza, lubricación, cohesión y paciencia del cuerpo. Respecto a los deshechos ( $m a-$ la), sudor, excremento, orina, su principal función es la de regular su flujo, mientras que con relación con la formación de los tejidos corporales (dhätu), su cometido es nutrir, animar, lubricar, colmar y desarrollar el embrión ${ }^{51}$.

Así, los doṣa se encuentran presentes en las primeras fases de la concepción del individuo, en el śukra o «semilla masculina» y en el ártava o «semilla femenina». De su unión surgen los tres tipos de constitución (prakrti): débiles (hina), medias (madhya) y poderosas (uttama). «El fuego digestivo y la predisposición intestinal son dos de los rasgos más significativos de los muchos que componen la constitución personal (prakrti) de un individuo» ${ }^{52}$. A diferencia del sāṃkhya, en el ayurveda el término prakṛti se refiere a las tendencias innatas, tanto mentales como físicas, características

\footnotetext{
50. CS 3. 6. 13, p. 190.

51. AH 1. 11. 1-29, p. 154.

52. Svoboda, n. 27, p. 76.
} 
de cada individuo. De las posibles combinaciones de los humores emergen siete constituciones diferentes, tres de cada una de las doșa por separado, tres de combinaciones de pares de doșa y una séptima en la que participan las tres en equilibrada proporción. La primera es débil, la segunda media y la tercera es la óptima o ideal ${ }^{53}$.

Un exceso de vāyu produce enflaquecimiento, oscurecimiento de la piel, temblores, estreñimiento y dificultad de evacuación. Los síntomas son una disminución en la percepción, la fuerza y el sueño, así como parloteos, mareos y abatimiento. Un incremento de pitta amarillea la piel, los ojos, la orina y los excrementos. Produce además hambre, sed, ardores e insomnio. Un aumento de kapha produce debilidad y pesadez, exceso de salivación y reducción del fuego digestivo. Los síntomas son palidez, frialdad y flojera en los miembros, produciendo resuello, tos y somnolencia.

Cuando los humores disminuyen, los síntomas son lógicamente otros. Una disminución de vāyu produce fatiga, mutismo, pereza y conciencia confusa. En este punto, las dolencias asociadas al aumento de kapha comienzan a manifestarse. La reducción de pitta disminuye las capacidades digestivas, produce frío y hace perder el buen aspecto. Cuando disminuye kapha los síntomas son mareos y vértigos, se vacían los receptáculos de la mucosidad, el corazón se acelera y las articulaciones se debilitan.

Vemos pues que la influencia de los humores en el organismo depende tanto de su cantidad (pramāna), como de sus cualidades (guna) y actividad $(\text { karma })^{54}$. Al estar presentes en cada uno de los tejidos del cuerpo, en cada momento del día y de la noche, tienden a concentrarse en aquellos lugares en los que es necesaria su actividad de redistribución de las energías vitales. Cuando se encuentran en su estado «normal» (avikrta) desarrollan y mantienen las diferentes funciones del organismo, pero tienen tendencia a degenerar (vikrta), incrementando ( $v r d d h i)$ o disminuyendo (kșaya) tanto en

53. Un detallado análisis de la caracterología humoral en Dash, n. 6, p. 37-45; Majumdar, n. 3, p. 44-47.

54. La referencia a los guna introduce el componente ético en la terapia ayurvédica. Todas las sustancias se encuentran formadas, en mayor o menor cantidad, por los tres guna y el balance entre estos determinará tanto la disposición psíquica como las inclinaciones físicas del individuo. Para un estudio detallado de los aspectos éticos en el ayurveda. Desai, Prakash. Medical ethics in India. Journal of Medicine and Philosophy. 1988; 13: 231-255. A este respecto véase también el trabajo de Weiss, Mitchell. Caraka Samhitā on the doctrine of Karma. In: Doniger, Wendy, ed. Karma and rebirth in classical Indian traditions. Berkeley: UCP; 1980, p. 90-115. 
cantidad como en cualidad o actividad. Es entonces cuando se corrompen los tejidos donde desarrollan su actividad, lo que puede desencadenar el colapso del organismo.

\section{Asiento y funciones de los humores}

Caraka ofrece un informe detallado de los lugares particulares que ocupan cada uno de los humores, aunque en mayor o menor medida se encuentren distribuidos por todo el organismo. El conocimiento de estos «asientos» es importante para las terapias de purificación. Respecto a su localización específica en el cuerpo, Majumdar ofrece tablas ilustrativas sobre sus asientos «propios»y otros lugares donde pueden encontrarse ${ }^{55}$. Sobre estas localizaciones los diferentes compendios no se ponen de acuerdo. Para Caraka el duodeno (ämäsaya) es asiento de pitta y kapha, mientras que para Suśruta es únicamente el asiento de kapha. Es probable que para Caraka àmāsaya haga referencia tanto al estómago como a la primera porción del intestino delgado, mientras que para Suśruta se refiere únicamente al estómago. Vāta se localiza principalmente en el intestino delgado, y también en la vejiga, el recto y los huesos de las piernas. El lugar natural de pitta es el estómago, aunque también circula por la sangre y los poros de la piel. El lugar de mayor concentración de kapha es el pecho, aunque también se mueve por la cabeza, las articulaciones y la grasa. La obra lista un total de ochenta dolencias asociadas a väta, cuarenta a pitta y veinte de kapha, enumerando los correspondientes con la perturbación de cada uno de los humores ${ }^{56}$.

Vāgbhata coincide con Caraka en que los tres humores impregnan todas y cada una de las partes del organismo y que, cuando no están corrompidos, se localizan en ciertas partes del cuerpo donde realizan funciones específicas para su mantenimiento. De ahí que se hable del «asiento natural» donde se concentran habitualmente. La esencia nos ofrece nuevos detalles sobre dichos «asientos». El asiento de vāta se encuentra en el intestino grueso (pakvāśaya), la cintura, los muslos, las orejas, los huesos y la piel. Aunque de todos estos lugares el principal es el intestino grueso. El ombligo, el

55. Majumdar, n. 3, p. 50-53.

56. CS 1.20.11, p. 363. 
estómago, el intestino delgado, la sangre, los ojos y la piel son los asientos naturales de pitta, aunque especialmente el nābhi, la región que rodea el ombligo. Por último, el pecho, la garganta, la cabeza, las junturas de los huesos, la grasa, la nariz y la lengua son los asientos naturales de kapha ${ }^{57}$.

El humor vāta o vāyu se subdivide en cinco tipos de «aires» que recorren el organismo realizando diferentes funciones. Como señala Majumdar, hay un único vāyu que adquiere diferentes nombres debido a las diferentes funciones que realiza en las diferentes partes del cuerpo. ${ }^{58} \mathrm{El}$ primer tipo, prāna, se localiza en la cabeza y se mueve a través del pecho y la garganta. Sirve de soporte a la mente, el corazón, los órganos sensoriales y la inteligencia. También cumple sus funciones en las expectoraciones, los estornudos, lo eructos, la respiración y en la función de tragar alimentos o bebidas. El segundo, udāna, se localiza en el pecho y se mueve a través de la nariz, el ombligo y la garganta. Es el fundamento de la facultad del habla, del entusiasmo, el color, la memoria y la capacidad de trabajo, juega un papel fundamental en cualquier tipo de esfuerzo físico. El tercero de los aires, vyāna, se localiza en el corazón pero se mueve a través de todo el cuerpo a gran velocidad. Realiza su contribución en actividades muy diversas como caminar, agachar o levantar partes del cuerpo, y abrir y cerrar los ojos. El cuarto, samāna, se localiza muy cerca del fuego digestivo y se mueve a lo largo de los conductos alimentarios, mantiene la comida en éstos durante un tiempo y ayuda en su "cocción» o digestión, separando en la sangre los desperdicios y ayudando a eliminarlos. Y finalmente, el quinto, denominado apana, se localiza en el intestino grueso y se mueve a través del pecho, la vejiga y los genitales, participando en la eyección del semen, la eliminación de los fluidos menstruales, de las heces y la orina. Asimismo, juega un importante papel en la extracción del feto ${ }^{59}$.

El segundo de los humores, pitta, es a su vez de cinco clases. La primera de ellas se denomina pācaka pitta (también anala: fuego), se encuentra localizada en el interior del intestino grueso, el intestino delgado y el estómago. Aunque se compone de los cinco elementos, tiene principalmente las cualidades del fuego (tejas) y, aunque es un fluido, carece de la viscosidad y de otras de las cualidades de los líquidos. Su principal función en la digestión y transformación del alimento. Se dice que «cocina» el ali- 
mento, separando la esencia que asimilará el cuerpo de lo que desechará. El segundo tipo de pitta se denomina rañjaka y se localiza únicamente en el estómago (āmáśaya). Debe su nombre a que tiñe de rojo las sustancias asimiladas (rasa). El tercer tipo se denomina sādhaka y se localiza en el corazón. Atiende a las funciones mentales (recordemos que el ayurveda sitúa la mente en el corazón), como el conocimiento, la inteligencia, la reflexión y la conciencia del yo. Un cuarto tipo de pitta se localiza en los ojos y se denomina ālocaka pitta porque ayuda a percibir la forma de los objetos. Por último, hay un pitta de la piel, denominado bhrākaka, que le confiere su color característico ${ }^{60}$.

El tercer humor, kapha, también realiza cinco funciones diferentes. El primero de ellos, denominado avalambaka kapha, se localiza en la zona de encuentro de la espalda, el cuello, el pecho y los hombros. Dado que tiene las cualidades del agua (cohesión, suavidad, fluidez y humedad), su tarea es la de mantener y dar cohesión al cuerpo, contribuyendo a fortalecer otros lugares de actividad de kapha. El segundo se localiza en el estómago y se denomina kledaka, pues humedece el bolo alimenticio. En la lengua se localiza el bodhaka kapha, que ayuda a la percepción del gusto. En la cabeza, el tarpaka kapha, que colabora en la percepción sensorial. Por último, el śleșaka kapha lubrica las junturas de huesos y articulaciones ${ }^{61}$.

Otra de las taxonomías en torno a los humores hace referencia a los diversos tipos de temperamento. Tanto Caraka como Suśruta describen en detalle las características mentales del individuo en función de los doṣa, así como las relaciones entre la caracterología humoral y el destino del individuo, ofreciendo un análisis detallado de los condicionamientos mutuos entre los humores y el karma de la persona ${ }^{62}$. Los tres principios biorreguladores forman el carácter básico de cada individuo, siendo el doṣa dominante es el que determina el carácter psicosomático de la persona ${ }^{63}$. Los compendios se preguntan si son estos agentes independientes o se encuentran de alguna manera supeditados al karma. Se responde que ambos corren en paralelo, sin que ninguno de ellos se encuentre condicionado por el otro. La mente se corresponde con el cuerpo y el cuerpo con la mente, la disminución de vāta supone una disminución del entusiasmo en la persona afectada y un

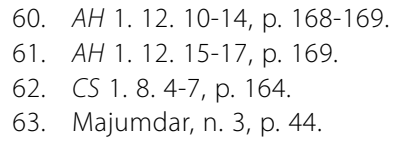


aumento de la tristeza, comenta Cakrapāni ${ }^{64}$. Así como hay tres humores corporales (śārīradoṣa) que causan la corrupción del cuerpo, hay también dos humores mentales, que son responsables de la inquietud y estupor de la mente. El primero de ellos, llamado rajas, se manifiesta en el orgullo, la impaciencia, la sensualidad y la ira. El segundo, tamas, mediante la pereza, la inercia y la condición obtusa. En ambos tipos de degradación debe buscarse la causa de las enfermedades internas ${ }^{65}$.

\section{Conclusiones}

En sus observaciones finales a la primera sección del Caraka, Cakrapāṇi ofrece una síntesis de lo que la ciencia del ayurveda supone y enseña. El cuerpo humano se encuentra compuesto por los cinco elementos. Sus diferentes funciones se mantienen gracias a una adecuada alimentación y una adecuada experiencia sensible, siendo los errores de juicio (prajñāparädha) y los cambios estacionales las principales causas de la enfermedad. La ruptura del equilibrio de los dhätu es responsable de las diferentes dolencias, pero también hay que tener en cuenta factores como el espacio y el tiempo. La administración de los tratamientos depende del momento y del lugar, no sólo con respecto a los hábitos de los diferentes pueblos, sino también con relación a la disponibilidad de los recursos terapéuticos ${ }^{66}$. Una observación que supone el reconocimiento de factores antropológicos en la enfermedad y de cómo el tratamiento médico puede también dar lugar a conflictos éticos ${ }^{67}$.

El crecimiento del cuerpo se basa en un principio analógico. Los tejidos corporales se desarrollan cuando el individuo se alimenta de sustancias que comparten los constituyentes de éstos y decaen cuando tienen las cualidades opuestas. La ciencia del ayurveda consiste precisamente en el conjunto de prescripciones (alimenticias, médicas y de conducta) que llevan

64. CS 1. 18. 52, p. 346 .

65. CS 4. 4. 34, p. 405.

66. Cakrapāni, comentario a CS 1. 30. 32, p. 604.

67. En una sociedad tan ritualizada como la india, la injerencia de las prácticas médicas en la actividad ritual ofrece un amplio campo para los estudios sociales de la ciencia. El tema de las relaciones entre el ayurveda y el dharma ha sido tratado, entre otros, por Wujastyk, Dominik. Medicine and Dharma. Journal of Indian Philosophy. 2004; 32: 831-842; Desai, n. 54, y Engler, Steven. Science versus religion in classical Ayurveda. Numen. 2003; 50: 416-463. 
al mantenimiento del equilibrio de los doșa o a recuperar dicho equilibrio cuando éste se ha perdido. Pero mantener la salud no sólo requiere de una dieta apropiada y una medicación correcta. El comportamiento (mental, sensorial y discursivo) juega también un papel fundamental y estos otros aspectos extradisciplinares darán lugar a numerosas tensiones entre médicos, filósofos y ritualistas. Se admite además que la enfermedad puede producirse a causa de un uso deficiente de las capacidades intelectivas y perceptivas, infrauso (ayoga), sobreuso (atiyoga) y abuso (mithyāyoga), usos deficientes que influyen en la gestión y equilibrio de los doṣa, lo que sitúa a los humores en un debate más amplio en el que participarán médicos, filósofos y legisladores de la tradición brahmánica ${ }^{68}$.

El presente artículo ha rastreado las diferentes concepciones de la doctrina humoral en el seno de las tres principales enciclopedias del periodo clásico, tratando de mostrar las diferentes divergencias existentes tanto en el seno de un mismo compendio, como es el caso de Caraka, como entre las enciclopedias posteriores de Suśruta y Vāgbhața. Caraka define la ciencia médica como «el conocimiento que facilita el equilibrio de los constituyentes del cuerpo» ${ }^{69}$, pero, como esperamos haber mostrado, las taxonomías de dichos constituyentes difieren no sólo entre los diferentes compendios sino también dentro de una misma enciclopedia. En algunos pasajes del Caraka encontramos referencias a siete $d h \bar{a} t u^{70}$, mientras que en otras secciones se listan como constituyentes únicamente vāyu, kapha y pitta $^{71}$, que llegan incluso a considerarse elementos permanentes en todos los cuerpos animales ${ }^{72}$, siendo el objeto de la medicina advertir cuando se encuentran en estado natural y en estado de irritación. Incluso hay pasajes de esta obra que incluyen los doșa entre los residuos (mala) cuando éstos no han sido todavía evacuados ${ }^{73}$, como ocurre en los textos del vinaya

68. Los tres compendios clásicos constituyen una rica fuente de información histórica sobre los aspectos sociales de la medicina en la antigüedad india. Basham, Arthur. The practice of medicine in Ancient and Medieval India. In: Leslie, Charles, ed. Asian medical systems: A comparative study. Berkeley: UCP; 1976, p. 21-39. Aunque su postura ideológica esté un tanto anticuada, otro estudio ilustrativo sobre los aspectos sociales de las prácticas médicas es el de Debiprasad, Chattopadhyaya. Science and society in Ancient India. Calcutta: Research India Publications; 1977, p. 188-201.

69. CS 1.1.53, p. 39.

70. CS 4. 6. 10, p. 432.

71. CS 1. 7. 39-41, p. 154-156.

72. CS 1. 18. 48, p. 346.

73. CS 1. 28. 4, p. 567. 
budista. Como hemos mostrado, el planteamiento de Suśruta presenta ciertas innovaciones respecto al de Caraka, fundamentalmente en que se proponen terapias para el fortalecimiento de los humores ${ }^{74}$ (Caraka sólo habla de calmarlos), y en el hecho de que los doṣa se consideran siempre constituyentes (dhātu) del cuerpo humano, ofreciendo las listas de órganos donde se localizan los humores cuando no se encuentran irritados ${ }^{75}$. A esta lista de tres Suśruta añade la sangre, que como causa de la enfermedad realiza un papel de intermediario ${ }^{76}$. Vāgbhața, el tercero en la triada de las autoridades médicas de la tradición sánscrita, presenta la forma definitiva de la teoría de los humores. Los tres doṣa son elementos presentes de manera constante en el cuerpo, su equilibrio supone la salud (doṣa-sāmya), su desequilibrio, la enfermedad (doșa-vaișāmya $)^{77}$, reemplazando la definición de la salud como dhātusāmya y de la enfermedad como dhātu-vaișāmya. El desarrollo semántico del concepto de doșa en el norte de India durante el periodo clásico, que primero significó dolencia patológica de vāyu, pitta y kapha y posteriormente fue un término común para designar estos tres componentes del cuerpo, podría explicar algunas de las divergencias expuestas en el presente artículo ${ }^{78}$. 


\section{ARTICLES}

\section{Divergences around humours in Sanskrit medical compendia}

Juan Arnau Navarro. ......................................

1.- Introduction. 2.-Substances and flavours. 3.-Humoural correspondences. 4.-Divergences around humours 5.-Localisation and functions of the humours. 6.-Conclusions.

ABSTRACT: This article traces the genealogy of divergences around humours in the Indian medical literature of the classical period, using the Sanskrit editions of the medical encyclopaedias of Caraka, Suśruta and Vāgbhața as primary sources. The basic thesis is simple: Indian medical traditions were developed within the framework of a theory of the humours, but the ancient sources of the Ayurvedic tradition show that there was never full agreement on the nature and functions of the three basic humours (vata, pitta and kapha). Some considered humours to be physical entities with a specific localisation in the body, while others regarded them as hypothetical entities for the classification of diseases that must be balanced by medical treatment. This ambiguity in their definition, delimitation and application leads to different ways of understanding the human body and its therapies. 